\title{
Prototype for a Personal Safety Gadget using Arduino Uno
}

\author{
Fathimath Zuha Maksood \\ L4 BSCO \\ Caledonian College of \\ Engineering \\ Sultanate of Oman
}

\author{
Moza Saif Al Yarubi, \\ Amani Salim Al Dhouani \\ L3, L4 BSCO \\ Caledonian College of \\ Engineering \\ Sultanate of Oman
}

\author{
Geetha Achuthan \\ Senior Lecturer \\ Caledonian College of \\ Engineering \\ Sultanate of Oman
}

\begin{abstract}
Personal safety has grown to become one of the most important requirements in today's world where children are abducted, women are threatened, and senior citizens manipulated. Increase in usage of technology and smart electronics provides an opportunity for a simple cost-effective safety gadget that helps the victims during unforeseen dangers. A successful attempt has been made to design and implement a prototype for an electronic gadget which has the potential to serve as a safety wear in the coming years. This paper covers descriptive details about the procedure, implementation, testing and results that were obtained. It also outlines the plans regarding the future plan and its potential release in the market. The design is microcontroller oriented and uses Arduino Uno and SIM900 GSM modem as its major components.
\end{abstract}

\section{General Terms}

Hardware, Microcontroller, Personal Safety, Computer Science, Electronics

\section{Keywords}

Arduino, SIM900 GSM Modem, LDR, Circuit

\section{INTRODUCTION}

Insurgent breakouts in the field of information technology can be relatively presumed as the best milestone achieved in the $21^{\text {st }}$ century. Usage of smart phones for various kinds of communication like e-mailing, chatting, SMS (Short Message Service) and MMS (Multimedia Service) services has increased the number of its users rapidly. Even though the roots of communication have visibly been brought to man's fingertips, recent years have witnessed personal safety as one of the most challenging issues in the world. In case of unforeseen dangers like abduction, medical emergencies, etc., there is a growing requirement for easy-to-use personal safety equipment for the community. Although rise in the number of crimes committed has created chaos and concern, gadgets that can guarantee safety have not received any spotlight. Current studies have suggested the existence of technologically advanced devices and applications in the field of personal safety, but none of these are known or fully functional in Oman. Hence, this paper attempts to design and develop a prototype for a personal safety gadget by utilizing the GSM modem and other existing technological resources with an aim to serve and support the residents with peace and the safety in Sultanate of Oman. The design involves simple components which are available in the local market.

\subsection{Purpose and Scope}

The following objectives have been precisely formulated:

- To design and develop a prototype for an easy-to-use personal safety gadget

- $\quad$ To employ Arduino Uno powered with ATmega328P as the microcontroller for the gadget

- To integrate Arduino circuit board with a SIM900 GSM Modem

- To send alerts to a specified set of mobile phone numbers using light as the stimulus

The scope of the prototype spans over the need for personal safety gadgets in hostile environments. It has the ability to bring a good impact on the community and encompasses various dimensions like programming, hardware designing, and most importantly, attempts at enhancing social welfare. Furthermore, a low cost mechanism for timely and needful communication is ensured. The prototype has been created based on this insight and it expects to reduce the intensity of crime related activities and provide an easy preventive measure to the victims.

\subsection{Description}

This system incorporates a schematic prototype for a potential hardware device which is designed to function only for security and safety purposes. Electronic gadgets usually are drained off their battery limits due to continuous usage. Hence, a circuit is made using Arduino uno circuit board integrated with ATmega328P microcontroller, a GSM modem, a light dependent resistor and connecting wires. The idea is to shed light on (or expose) the resistor so as to trigger the microcontroller to run the program activating the call and sending the pre stored message to the recorded number/s using the GSM modem. This hardware mechanism has the potential to be used as premier product promoting the need for security and safety in growing urban localities.

\section{LITERATURE REVIEW}

\subsection{Safety and Security Threats and their Preventive measures}

"Technological progress is like an axe in the hands of a pathological criminal", quoted Albert Einstein in late 1917. With the passage of almost a century and faster evolution in the field of technology, one would certainly exclaim at the intensity of diverse incidents of crime that have taken place since then. Prevention of crimes and hazards form a major part in the development of a nation. Physical abuse, hate 
crimes, aggravated assault, resulting in serious injuries, threats and emotional attacks are some of the crucial yet dangerous types of crimes existing in the present era [1]. A country's jurisdiction is responsible for regulating these felonies and engaging the criminals for their committed crimes. But, the present-day society is characterized by highly publicizing these crimes on media or on the internet, informing other people about the existence and trials of criminals, generating hate and confusion in the community [2]. Even though publicity has its effects of collectively notifying a huge group of people, it does not necessarily instill the feeling of safety and security on them. Rather, it creates doubt and uncertainty and reduces faith in the legal bodies of the nation. Apart from unnatural hazards and crimes, situations of emergency can arise in case of health such as heart attacks, seizures, stroke and fainting. Seriousness of medical issues are often directly proportional to age and severity of the illness in each patient; but, unpredictable and acute events can be dangerous and lead to death.

\subsection{Safety and Security Measures in Local Region}

Sultanate of Oman is considered to be a nation well acclaimed by tourists and expatriates due to the feeling of safety and security it instills on its citizens. Although, the number of reports dealing with death due to crimes, hazards and medical emergencies are marginal, the crime rates of the country have risen from low to medium in the past few years [3]. Prevention of crimes in the region is a job well executed by various departments under the Directorate General of Inquiries and Investigations including the directorates of crime control, narcotics control, criminal laboratory, criminal evidence, quality control, etc. [4]. Even though crimes in Oman can be narrowed down to crimes of opportunity and petty theft, physical assault and manslaughter have been reported beginning with the past few years. Emergency contact number is the fastest and easiest method used by people during danger and situations involving crime. As easy as it is to remember and dial the number during unanticipated scenarios, the victim might not be able to get in touch with a phone or a telecommunication service of any form. There is no convenient or go-to strategy which can be employed during such situations. As mentioned, a series of confusion is generated following the occurrence of criminal related circumstances; but, execution of productive strategies is very less in order to console and deal with the victims and help them overcome similar forthcoming scenarios. Methods of curing the aftermath of crimes are focused upon, while ways to eradicate them in the first place are very few and of concern. Therefore, situational awareness and emergency contacts in case of suspicion are the best methods expected to be followed in Oman during crime related situations.

\subsection{Security Systems using GSM Communication Module and Arduino}

Since its initial release, Arduino programmable circuit boards have grown widely in terms of reliability, ease of use and compactness. Projects are occasionally set up by interfacing Arduino boards with a wide variety of electronics including, LCDs, GSM shields, etc. Data Acquisition and Home automation systems [5] have been developed by interfacing powerful microcontrollers with hardware components confirming its validity and usability spanning many areas. There have been attempts to promote security measures with the usage of these components. Due to the demanding maintenance of expensive CCTV cameras in an average home, people have begun to look out for cost-efficient solutions for security. This gave rise to the development of an advanced low-cost security system using GSM communication module [6]. Thermal, passive infrared and proximity sensors along with Arduino Uno and a SIM300 module are used to ensure safety and security. The operation mechanism involves the three sensors continuously detecting the change in stimulus i.e., the thermal sensor detects the change in temperature by looking out for fire related hazards, the passive infrared sensors take advantage of the pyro electricity to detect a human body which is a constant source of radiation, while the proximity sensors at the doors and windows help in detecting the presence of intruders or burglars. These sensors trigger the alarm and communicate with the microcontroller board to send text messages to the owner's mobile phone using the GSM modem, thereby, enhancing security using a cost efficient system.

\subsection{Proposed System}

The growth in technology in the country is used to formulate a solution which can provide a sense of security for the people residing in the nation. Thus using the knowledge gained from the survey and self-study an a hardware prototype is designed and developed by interfacing Arduino Uno with SIMCOM produced SIM900 GSM modem and using an LDR as the receiver for stimulus. Users are expected to place the hardware in the dark and only expose it when faced with unforeseen hazards. The intensity of light will trigger the circuit board which in turn powers the GSM modem to send text messages to the victim's emergency contact numbers.

\section{DESIGN AND TESTING 3.1 Design Purpose}

The purpose of proposed hardware design is to assist a personal safety application that can uniquely feature the importance of safety and security apart from securing space for the sole purpose of running the application. At present, people, especially young women tend to carry objects which apparently help defend themselves during unpredicted situations. These include pepper spray, light boots, belts and stun guns. Even though the assistance from these objects can provide relief, it is necessary that a victim can handle a bigger situation and can call out for help without being noticed. In these cases, it is always necessary to be swift and witty and produce an action which would blind the source of danger and press for help.

A potential hardware plan was formulated with a long term perspective which involved a wrist band, digitally controlled by a micro controller and programmed to make phone calls as well as send SOS messages when required. The stimulus for the entire system is chosen to be light and hence a light dependent resistor acts as the medium allowing the Arduino to trigger the messages to the victim's emergency contact numbers.

\subsection{Components used}

The various components involved in the circuit designinclude:

Arduino Uno - Arduino Uno is a specially designed circuit board used for programming and prototyping driven by Atmel based microcontroller, ATMega328P. Being one among the most widely used platforms in the world, Arduino has an open source design and unlike previous circuit boards, do not require additional hardware to burn programs onto the board. 
This indicates that the codes written for the microcontroller can be loaded on to Arduino Uno using USB cables, which are easier to carry and deal with. As seen in the figure, the Arduino Uno is powered using ATmega328P microcontroller. The circuit board has a convenient power management which allows the input voltage up to $12 \mathrm{~V}$. The built-in power regulatory mechanism then permits the regulation of this voltage into $5 \mathrm{~V}$ and $3.3 \mathrm{~V}$. The power can be supplied by connecting an adapter to the external power supply or to computers (or other devices) using a USB cable. The figure clearly represents the existence of 14 digital I/O pins as well as 6 analog I/O pins. These pins are the circuit board's medium of communication to external devices. Apart from these features, Arduino Uno accommodates $32 \mathrm{~KB}$ flash memory for the storage of programs.

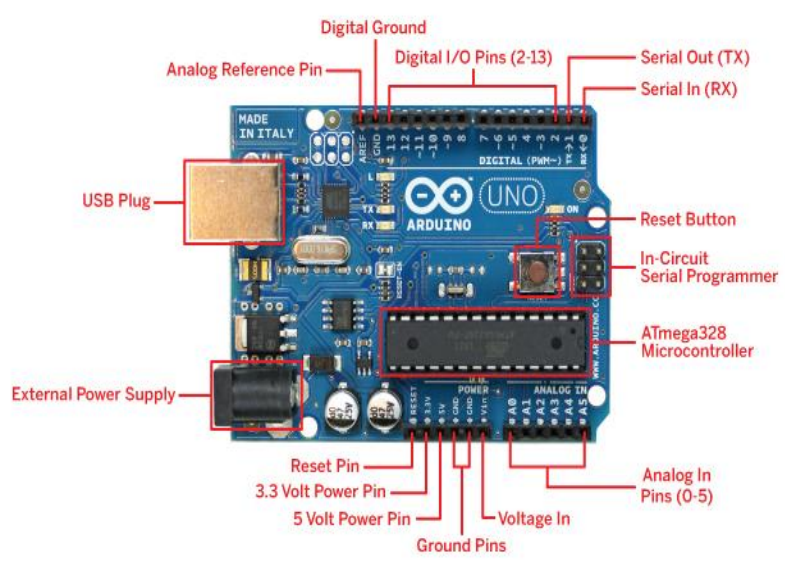

Figure 1 Arduino Uno [7]

Arduino packages include circuit board as given in fig. 1 as well as a piece of software, Arduino IDE (Integrated Development Environment). Since its introduction, many different versions of this software have been released and is basically used to code, compile as well as upload the programs onto the board. Arduino IDE uses a simplified version of $\mathrm{C}++$, providing an open source platform as well as various examples of code, resulting in Arduino rising to the most popular programmable circuit board series. Due to its functionality and efficiency, it is used in the design.

SIM900 GSM Modem - SIM900 is a quad band modem which is flexible to operate in a wide range of frequencies involving 850, 900, 1800, and $1900 \mathrm{MHz}$. It supports a differential range in order to be apt for worldwide compliance. Simcom produced SIM900 GSM modem which is shown in figure 2 has been used as it is ultra-compact, reliable and uses the powerful wireless SIM900 module. It has a powerful single chip processor and is accustomed to deliver clarity in voice, SMS, data and fax in a small form factor and with low power consumption. Since the work involves an exclusive functionality, we have employed a mini board with SIM900 modem and a GSM antenna. There are 16 pin positions soldered TTL connectors so as to easily interface it with microcontroller boards. Two LEDs are placed at each end of the circuit board to indicate the presence of power and network. A voltage of $5 \mathrm{~V}$ is required to power up the circuit board which and TX and RX pins are used for sending and receiving data. A network registered SIM card is required to activate the GSM modem.

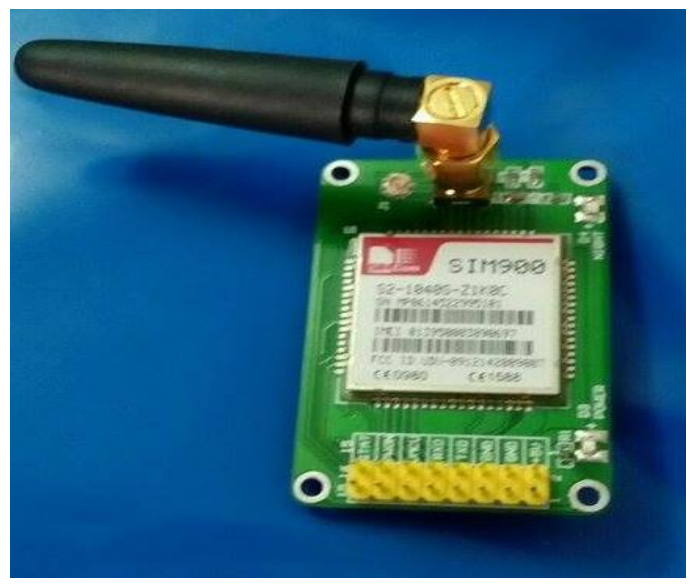

Figure 2 SIM900 GSM Modem

Various terminal soft wares exist in order to determine the correct functioning of a GSM modem, such as Terminal, SSCOM, etc. These modems are programmed using AT commands. Fig. 3 below depicts the different AT commands present.

\begin{tabular}{|l|l|}
\hline AT Command & The Functions of AT Command \\
\hline ATD & Dial \\
\hline AT+CGMS & Send SMS Message \\
\hline AT+CMSS & Send SMS Message from storage \\
\hline AT+CMGL & List SMS Messages \\
\hline AT+CMGR & Read SMS Messages \\
\hline$A T+C S C A ?$ & Service Centre Address \\
\hline AT+CPMS & To choose storage from ME or SM \\
\hline AT+IPR $=0$ & To choose auto baud rate \\
\hline AT + CMGF $=$ & To choose PDU Mode or Text Mode \\
\hline
\end{tabular}

Figure 3 List of AT Commands [8]

Apart from the two major components, a light dependent resistor (LDR) is used to resist the electricity using the intensity of light along with a $100 \mathrm{kOhm}$ resistor, breadboard and jumper cables (M-M and F-M).

\subsection{Block Diagram and Description} power

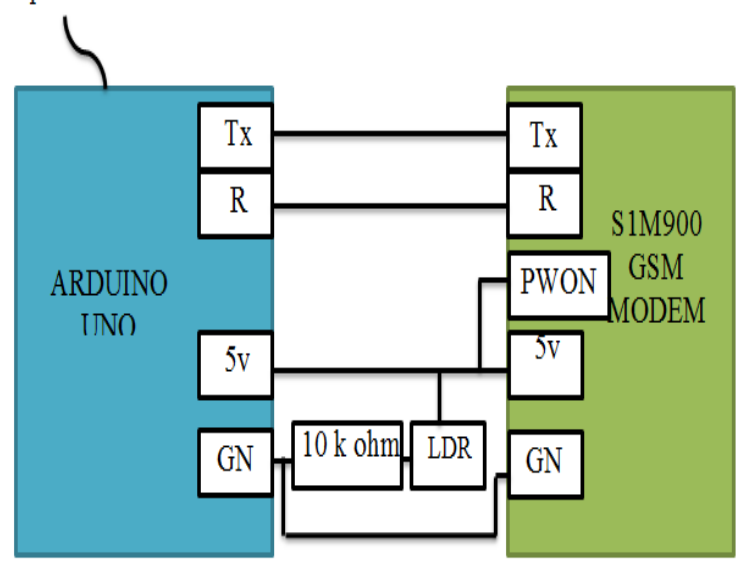

Figure 4 Block Diagram 
The aim of the designed circuit is to create a prototype which can utilize light as a stimulus and produce actions to alert people. The circuit, as stated, involves a series of components such as Arduino Uno, SIM900 GSM modem, photo-resistor, 100k resistor, breadboards and connecting jumper wires. The ATmega328P microcontroller acts as the power house while Arduino Uno in itself is powered via a USB cable. The power gained by the circuit board is regulated to $5 \mathrm{~V}$ and $3.3 \mathrm{~V}$ pins. The voltage in the $5 \mathrm{~V}$ pin is redirected to create a potential difference in the SIM900 GSM modem as well as the photoresistor. The second terminal of the Light Dependent Resistor is connected to the $100 \mathrm{k}$ resistor and the circuit is fully grounded. The main circuit connection lies in the transmission of data between the Arduino Uno and GSM modem, which requires the activation of TX (transmitter) and RX (Receiver) pins on both the circuit boards. According to the logical rule, TX of Arduino Uno is connected the RX of SIM900 GSM modem and vice versa.

\subsection{Operation of the Circuit}

The working of the electronic setup begins with the gain of voltage across the components that are connected to form the hardware design. The source of power in this case was the laptop battery sharing the potential with the Arduino Uno board. When the entire setup is brought to an "ON" mode, the LDR starts regulating the amount of light intensity that falls on it. The value of light intensity captured by the LDR is read by one of the analog input pins on the Arduino board, which is then compared to a threshold level. If the value goes beyond a predetermined level, the Arduino triggers the GSM modem to send a text message to the numbers which are programmed in the Arduino IDE. The SIM card placed in the GSM modem utilizes the network signal and immediately transmits the character data. The reset button should then be pressed in order to rerun the mechanism.

To summarize the above mentioned circuitry, a victim is expected to carry this device in a closed space (such as in a hand bag or in the pockets). When faced with an unprecedented situation or hazard, the victim just has to flash the device or expose it into the light. This can be done in a swift motion without attracting attention, all while triggering the GSM modem to send the required text messages. When used portably, the entire setup cane be powered using a battery which can then be replaced from time to time .Since the hardware is exclusively used as a safety gadget, it is not deprived or drained of charge due to unnecessary tasks.

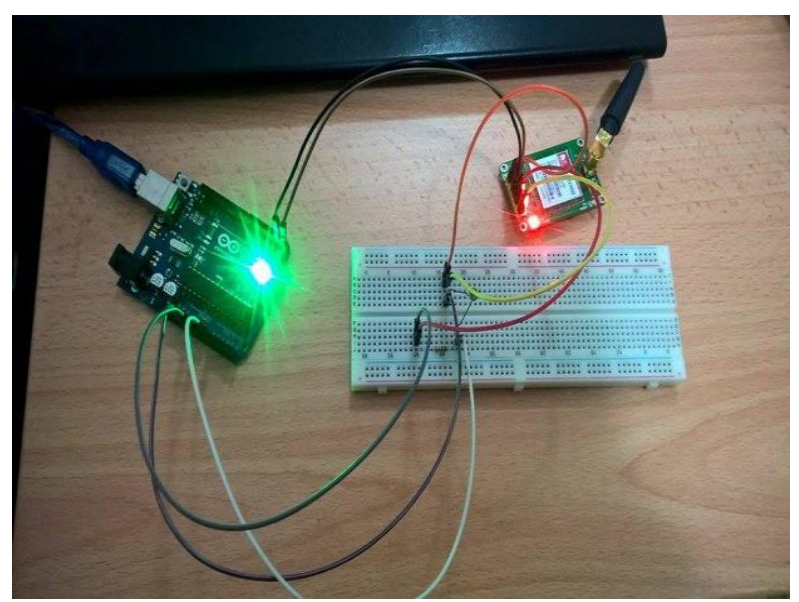

Figure 5 Circuit using Arduino and SIM900 GSM Modem

\subsection{System Flowchart}

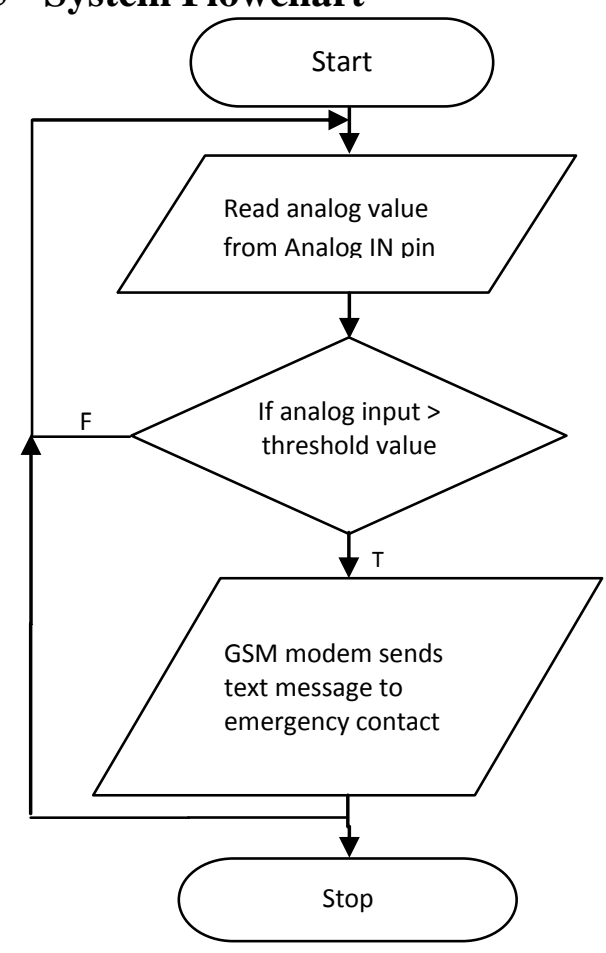

Figure 6 Flowchart of the System

\subsection{Implementation and Testing}

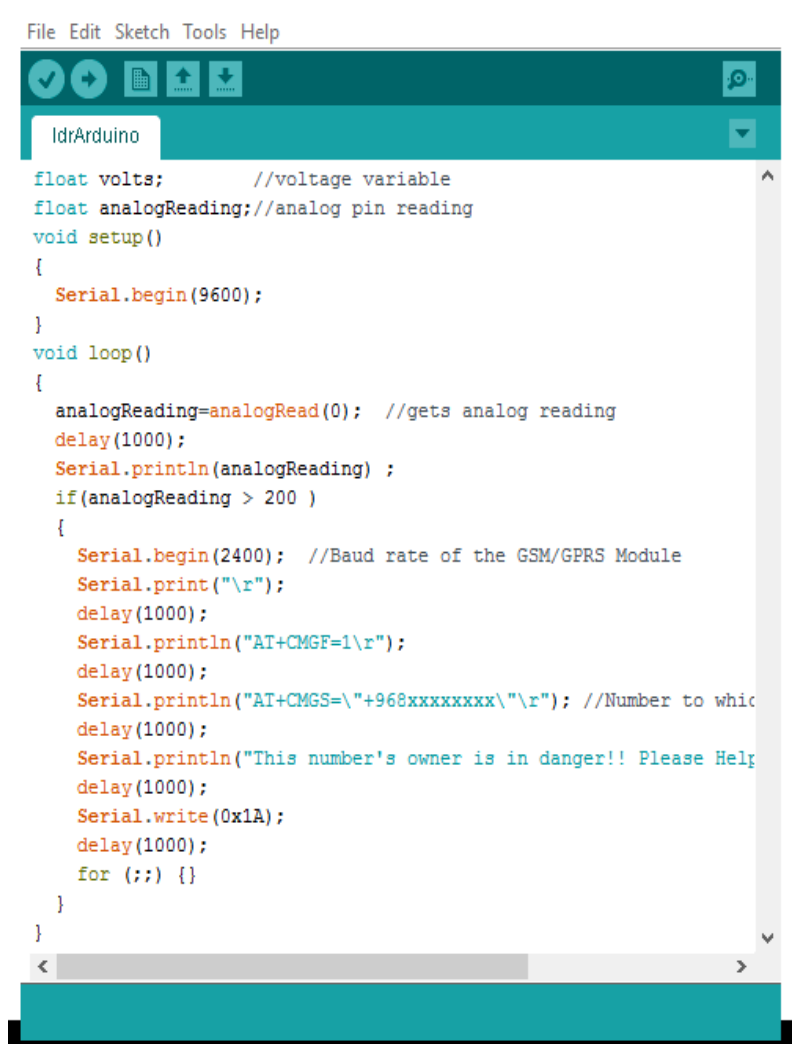

Figure 7 Program on Arduino IDE

A program was coded in Arduino IDE and burned on to the board via a USB cable. The methods used in the program involved: 
- $\quad \operatorname{analogRead}()$ - This function is used to read the analog input from the pin to which the LDR is connected. It renders the value which has to be compared to a threshold in order to receive the expected output.

- Serial.begin(baud rate) - It begins an interface with the Serial output of Arduino UNO which can be the serial monitor or any device connected to it. The baud rate determines the device to which the output is delivered.

- $\quad$ Serial.println() / Serial.print () - This is a default method which prints the output on the Serial monitor or to the device connected serially to the Arduino Board.

- delay(integer) - It creates a time delay of integer microseconds during run time.

- $\quad$ AT commands - AT commands are used to communicate and properly interface Arduino UNO with the GSM Modem.

The code also employs an IF conditional construct in order to compare the output value to the threshold limit. The Arduino IDE and the code for the hardware implementation are shown in Fig. 7.

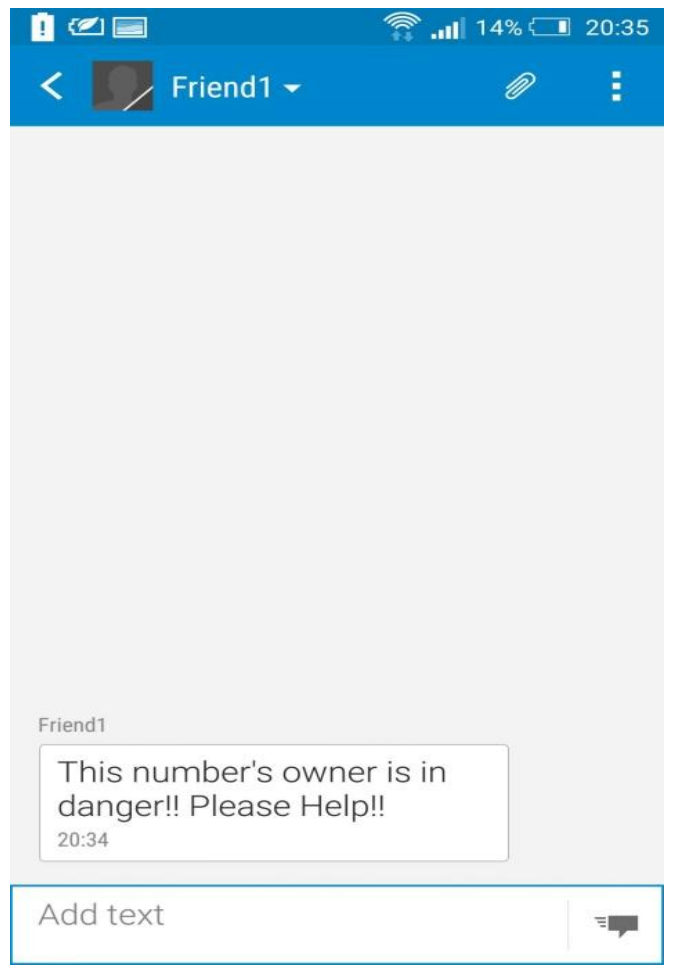

Figure 8 Message received on Phone

The stored message in the Arduino IDE is forwarded to the emergency contact numbers of the user and the message is delivered successfully as shown in Fig. 8. The hardware design was implemented with care and precaution as it dealt with programmable circuit boards which could burn off even with slight fluctuation in input voltage. The power was supplied via a USB cable using a laptop. An Ooredoo SIM card was inserted into the SIM card slot on the GSM modem in order to test the Short Message Service. The results produced were different from the expected due to unstable connections and after fixing the connections expected results are obtained. Terminal software is used to test the functionality of the GSM modem using AT commands. It is found that an extra connection had to be made to the "power on' pin in order to trigger a strong network connection. The LEDs present at two ends of the board had to blink in order to secure a perfect circuit. It is also found that the pin code of the SIM card has to disabled prior to its use in the GSM modem. Finally, the entire circuit has to be properly grounded in order to achieve expected results.

\section{CONCLUSION AND FUTURE WORK}

A hardware circuit is built using Arduino uno microcontroller, a GSM modem, a light dependent resistor (LDR) and connecting wires. The idea is to shed light on (or expose) the LDR so as to trigger the microcontroller to run the program activating the call and sending the pre stored message to the recorded number/s using the GSM modem. Testing and verification of the hardware was performed and the proper functioning is ensured. SMS is send successfully to the set of emergency numbers.

\section{Future Work}

The prototype developed can be introduced in the market in a wearable form so that people can use it under their top sleeves. The messages sent can be converted into local language for regional use and to reach out to more users in Oman. Furthermore, the upgraded GSM modem can be used in order to send the location as well as voice messages and calls.

\section{ACKNOWLEDGEMENTS}

We express our gratitude to The Research Council (TRC), Oman for providing us the opportunity and granting us the required support to accomplish this endeavor under FURAP. We are forever grateful to our institution, Caledonian College of Engineering Oman and the college executives for the wonderful opportunity to implement our ideas into reality.

\section{REFERENCES}

[1] Bureau of Justice Statistics, 2014. Terms \& Definitions: Crime Type. [Online]. Available from: http://www.bjs.gov/index.cfm?ty=tdtp\&tid=3. [Accessed: 20th June 2015].

[2] Shoener, N., 2015. What Are the Different Types of Crimes?[Online].Available from: http://www.legalmatch .com/law-library/article/what-are-the-different-types-ofcrimes.html. [Accessed: 20th June 2015].

[3] Bureau of Diplomaic Security, 2015. Oman 2015 Crime and Safety Report. [Online]. Available from: https://www.osac.gov/pages/ContentReportDetails.aspx? cid=17013. [Accessed: 21st June 2015].

[4] Royal Oman Police, n.d. Directorate General of Inquiries and Investigations. [Online]. Available from: http://www.rop.gov.om/english/dg_cid.asp.[Accessed: 21st June 2015].

[5] Jivani, M.N., 2014. GSM Based Home Automation System Using App-Inventor for Android Mobile Phone. International Journal of Advanced Research in Electrical,Electronics and Instrumentation Engineering[e-journal]. 3 (9).p. 12121 - 12128.Available from: http://www.rroij.com/open-access/gsm-basedhome-automation-system-usingappinventor-for-androidmobile-phone.pdf. [Accessed:2nd September 2015].

[6] Katara, P. et al., 2014. Advanced Low-Cost Security System Using Gsm Communication Module 
International Journal for Scientific Research \& Development[e-journal]. 2 (5).p. 53-54.Available from: http://www.ijsrd.com/articles/IJSRDV2I5044.pdf. [Accessed:4th September 2015].

[7] Arduino Uno .n.d [Online].Available from: http://thietbichetao.com/san-pham/kit-phat-trien/arduinouno-r3/. [Accessed:31st August 2015].

[8] Common AT command 2012 [Online].Available from: http://fyp2ain.blogspot.com/2012_03_01_archive.html. [Accessed:1st September 2015].

[9] Al-Jabri, K.S., 2007. Fire public awareness in Oman. Journal of Scientific Research and Essays[e-journal]. 2 (8).p.468-475.Availablefrom: http://www.academic journals.org/article/article1380187095_Al-\%20Jabri.pdf. [Accessed:21st June 2015].

[10] Anon., 2013. Five arrested in Sohar for kidnapping, rape.
Muscat Daily.[Online]. 23rd March. http://www.muscatdaily.com/Archive/Oman/Fivearrested-in-Sohar-for-kidnapping-rape-24lk. [Accessed:9th July 2015].

[11] Anon., 2013. Two arrested for kidnapping, rape in Oman. Muscat Daily.[Online]. 18th February. http://www.muscatdaily.com/Archive/Oman/Two-arrest ed-for-kidnapping-rape-in-Oman-21ta.[Accessed:8th July 2015].

[12] Jayakarthigeyan, 2013. GSM Modem with Arduino UNO.[Online] 7th February 2013.Available from: http://letsplaywitharduino.blogspot.com/p/gsm-modemwith-arduino-uno.html. [Accessed:30th August 2015].

[13] Timothy, 2014. Light-Dependent Resistor (LDR). [Online]. Available from: http://www.arduinohacks.com/light-dependent-resistor-ldr/. [Accessed: 2nd September 2015]. 